\title{
Transition Delay via Vortex Generators in a Hypersonic Boundary Layer at Flight Conditions
}

\author{
Pedro Paredes* \\ National Institute of Aerospace, Hampton, VA 23666, USA \\ Meelan M. Choudhari ${ }^{\dagger}$ and Fei $\mathrm{Li}^{\ddagger}$ \\ NASA Langley Research Center, Hampton, VA 23681, USA
}

\begin{abstract}
The potential of realizable, stationary streaks undergoing nonmodal growth to stabilize a hypersonic boundary-layer flow and, subsequently, delay the laminar-turbulent transition onset, is studied via numerical computations. The geometry and flow conditions are selected to match a relevant trajectory location from the ascent phase of the HIFiRE-1 flight experiment, namely, a 7-degree half-angle cone with $2.5 \mathrm{~mm}$ nose radius, freestream Mach number of 5.30, freestream unit Reynolds number equal to $13.42 \times 10^{6} \mathbf{m}^{-1}$, and wall-to-adiabatic temperature ratio of approximately 0.35 over most of the test article. This paper investigates flow modifications induced by wall-mounted vortex generators (VGs), followed by an analysis of the modal instability of the perturbed, streaky boundary-layer flow. Results are presented both for a single array of VGs that was designed on the basis of optimal growth theory and for a VG configuration involving two separate arrays with opposite orientations that ware designed to provide staged control of flow instabilities while simultaneously reducing the amplification of streak instabilities resulting from the control devices. Earlier research had shown that the onset of transition during the HIFiRE-1 flight experiment, which did not include any control devices, correlated with an amplification factor of $N=14.7$ for the planar Mack modes. If one assumes that the transition $N$-factor is not affected by the introduction of the VGs, then the control configurations based on a single array of VGs and two separate arrays would result in a transition delay of $17 \%$ and $40 \%$, respectively. These findings suggest a passive flow control strategy of using VGs to induce streaks that would delay transition in hypersonic boundary layers dominated by Mack-mode instabilities.
\end{abstract}

\section{Nomenclature}

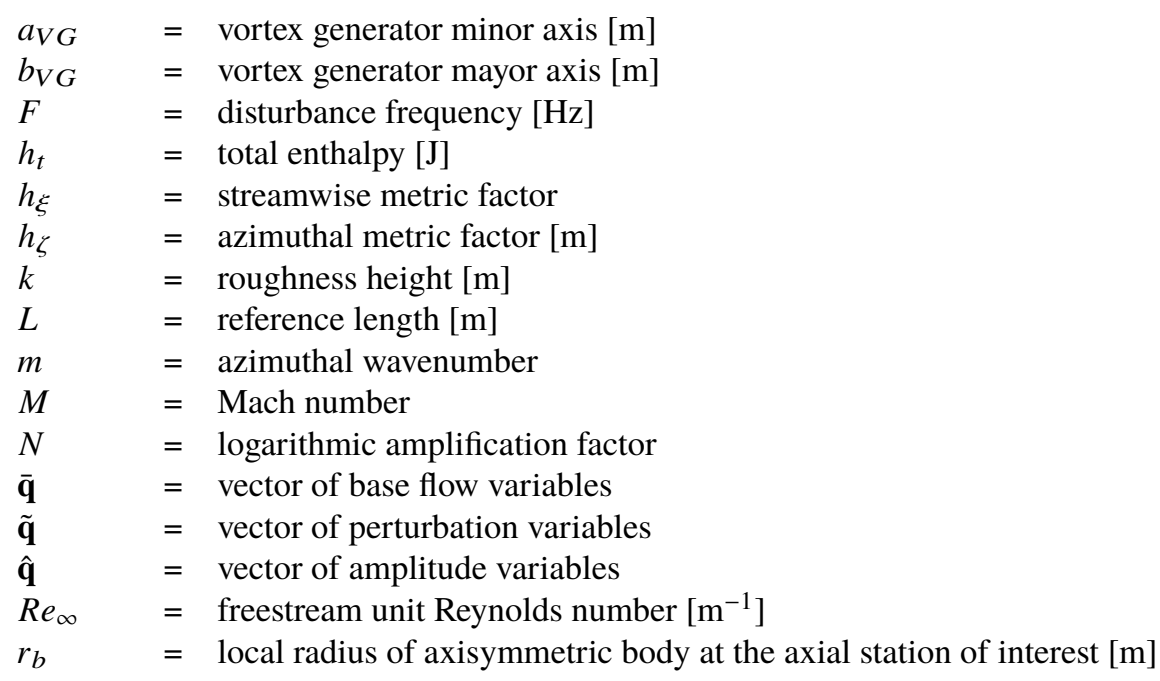

\footnotetext{
*Research Engineer, Computational AeroSciences Branch, NASA LaRC. AIAA Senior Member

${ }^{\dagger}$ Research Scientist, Computational AeroSciences Branch. AIAA Associate Fellow

${ }^{\ddagger}$ Research Scientist, Computational AeroSciences Branch.
} 


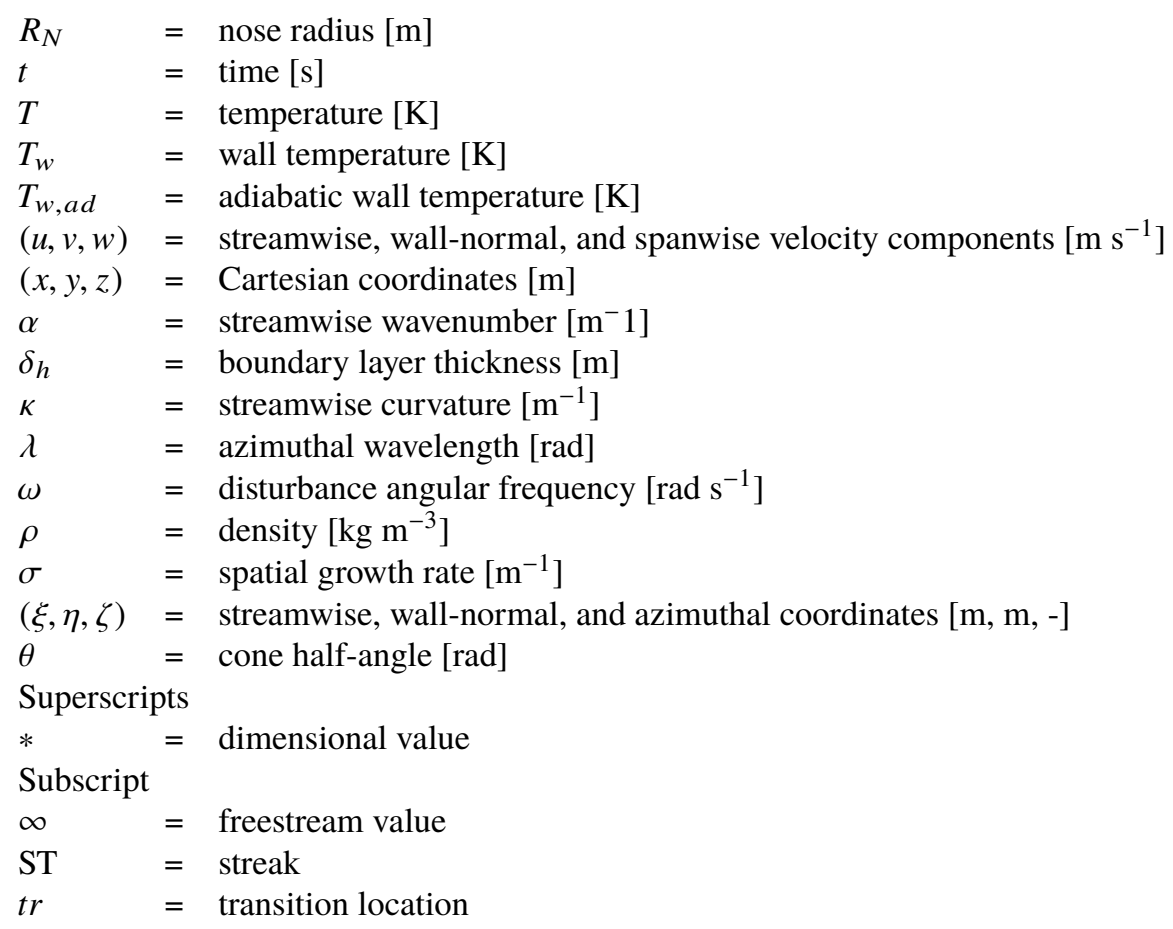

\section{Introduction}

Laminar-turbulent transition of boundary-layer flows can have a strong impact on the performance of hypersonic vehicles because of its influence on the surface skin friction and aerodynamic heating. Therefore, the prediction and control of transition onset and the associated variation in aerothermodynamic parameters in high-speed flows is a key issue for optimizing the performance of the next-generation aerospace vehicles.

Under low levels of background disturbances, transition is initiated by the exponential amplification of linearly unstable eigenmodes, i.e., modal instabilities of the laminar boundary layer. In two-dimensional boundary layers over sufficiently smooth aerodynamic surfaces, different type of instability mechanisms dominate the exponential growth phase depending on the flight speed. In the incompressible regime, the most amplified disturbances correspond to planar, i.e., two-dimensional, Tollmien-Schlichting (TS) waves, whereas oblique first-mode instabilities are dominant in supersonic boundary layers. The hypersonic regime is again dominated by the growth of planar waves of the second mode, i.e., Mack-mode type [1].

In the presence of sufficiently strong external disturbances in the form of either freestream turbulence (FST) or three-dimensional wall roughness, streamwise streaks involving alternately low and high streamwise velocity have been observed to appear in incompressible boundary layers [2, 3]. Further research in the incompressible regime has shown that streaks with sufficiently high amplitudes can become unstable to shear layer instabilities that lead to a form of "bypass transition" [4]. When the streak amplitudes are low enough to avoid transition due to streak instabilities, i.e., when the background disturbance level is relatively moderate, the streaks can actually reduce the growth of TS waves in incompressible flows as documented in both theoretical and experimental studies [5-7]. The stabilizing effect of stationary streaks in low-speed boundary layers has been used in passive flow control strategies to demonstrate transition delay via miniature vortex generators along the body surface [8, 9].

Numerous research efforts have focused on the tripping of hypersonic boundary-layer flows due to roughness elements. However, a few experimental and numerical studies have also reported a roughness induced delay in transition under specific circumstances. The latter studies have primarily focused on two-dimensional roughness elements. James [10] used fin-stabilized hollow tube models in free flight with a screw-thread type of distributed two-dimensional roughness. He found that for a given freestream Mach number between the range of 2.8 to 7 , there exists an optimum roughness height that yields the largest delay in transition. Fujii [11] studied the effects of two-dimensional roughness by using a $5^{\circ}$ half-angle sharp cone at a freestream Mach number of 7.1. He also observed transition delay under certain conditions when the wavelength of the wavy wall roughness was comparable to that of the Mack-mode instabilities. More recently, Fong et al. [12, 13] performed numerical and experimental studies, respectively, that were focused on 
the effect of two-dimensional surface roughness on the stability of a hypersonic boundary layer at a freestream Mach number of 6. The experiments [13], carried out in the Boeing/AFOSR Mach 6 Quiet Tunnel at Purdue University, used a flared cone model with 2D roughness strips and supported the numerical predictions indicating a stabilizing influence of roughness on the amplification of Mack-mode disturbances [12]. In particular, these studies showed that the most dominant Mack-mode instability could be suppressed via a judicious placement of the roughness elements along the cone surface.

Additionally, there is limited experimental evidence concerning delayed transition in a hypersonic boundary layer due to three-dimensional roughness elements. Experiments by Holloway and Sterrett [14] in the NASA Langley 20-inch Mach 6 tunnel used a single row of spherical roughness elements that were partially recessed within a flat plate model. Data for multiple values of the boundary-layer-edge Mach number were obtained by varying the plate mounting angle. These investigators found that, for cases with the smallest roughness diameters, transition was delayed for edge Mach numbers of larger than 3.7, which approximately corresponds to the lower bound for second-mode dominance over first-mode instabilities in a flat plate boundary layer at typical wind tunnel conditions. Therefore, their results are suggestive of a stabilizing influence of roughness-induced streaks on Mack-mode waves. Consistent with additional findings by Holloway and Sterrett [14], recent research [15, 16] has also established that the same streaks can develop high-frequency instabilities when the roughness height becomes sufficiently large, resulting in an earlier transition relative to that over a smooth surface.

Theoretical studies of the interaction between stationary disturbances and Mack-mode instabilities in hypersonic boundary layers were first initiated during the last decade. Li et al. [17] studied the interaction of Görtler vortices with Mack-mode instabilities on a flared cone, demonstrating a possible route to transition via this interaction. Li et al. [18] studied the secondary instability of crossflow vortices in the hypersonic boundary layer over a yawed cone and found that nonlinearly saturated crossflow vortices destabilize the Mack modes, which dominate the onset of transition in comparison with the intrinsic secondary instabilities of crossflow vortices, i.e., instability modes that do not originate from the second mode instability [19, 20]. Ren et al. [21] studied the stabilizing effect of weakly nonlinear suboptimal streaks and Görtler vortices on the planar first-mode and Mack-mode instabilities. They documented a slight reduction in the logarithmic amplification factor of approximately $\Delta N=0.2$ relative to the baseline, zero-streaks flat plate boundary layer. Additional investigations [22-24] related to axisymmetric, hypersonic boundary layers over circular cones in ground test facilities as well as under flow conditions representative of high altitude flight have shown that finite-amplitude optimal growth streaks can substantially reduce the amplification of planar Mack-mode instabilities, but also that oblique first-mode instabilities can be destabilized.

The development of roughness-induced streaks is strongly dependent on the details of roughness element shape, height, and spanwise or azimuthal spacing. The optimal growth theory provides a conceptually simple model to characterize the transient algebraic growth and subsequent slow decay of boundary-layer streaks due to an arbitrary initial disturbance; see Schmid [25] for a review. The transient growth arises as a result of the non-normality of disturbance equations, and the optimal growth theory seeks to maximize the disturbance growth across a specified streamwise interval. Regardless of the flow Mach number, the disturbances experiencing the highest magnitude of transient growth have been found to be stationary streaks that arise from initial perturbations that correspond to streamwise vortices. The instabilities of optimal streaks with finite initial amplitudes in supersonic and hypersonic boundary layers has been recently addressed by [26-28]. Paredes et al. [29] investigated the interaction of nonlinear stationary optimal growth perturbations with modal instabilities in a Mach 3 adiabatic flat-plate boundary-layer flow. At the selected flow conditions, the most-amplified linear waves correspond to oblique, first-mode instabilities. The analysis showed that optimally growing stationary streaks can destabilize the first-mode waves, but only when the spanwise wavelength of the instability waves is equal to or smaller than twice the streak spacing. Thus, as long as the amplification factors for the destabilized, short wavelength instability waves remain below the threshold level for transition, a significant net stabilization is achieved. On the other hand, the effect of nonlinear stationary streaks on the growth of Mack-mode instabilities has been investigated by Paredes et al. [24]. They demonstrated that the introduction of finite-amplitude optimal growth streaks in a Mach 5.3 axisymmetric flow over a cone at high-altitude flight conditions may lead to a notable delay in transition onset. Their results indicated that the planar Mack-mode waves were the most amplified instabilities for both the unperturbed and perturbed cases up to a threshold amplitude of the stationary streaks and that the subharmonic sinuous streak instability would dominate the transition process. However, the optimal growth streaks may not be easily realizable in practice, and the effects of realizable streaks due to wall-mounted roughness elements on the instability characteristics of a hypersonic boundary layer have not been studied as yet. The present work seeks to bridge that gap, with the goal of developing a more thorough knowledge base for transition prediction in the presence of realizable stationary streaks and expand the range of available techniques for transition control at hypersonic flight 


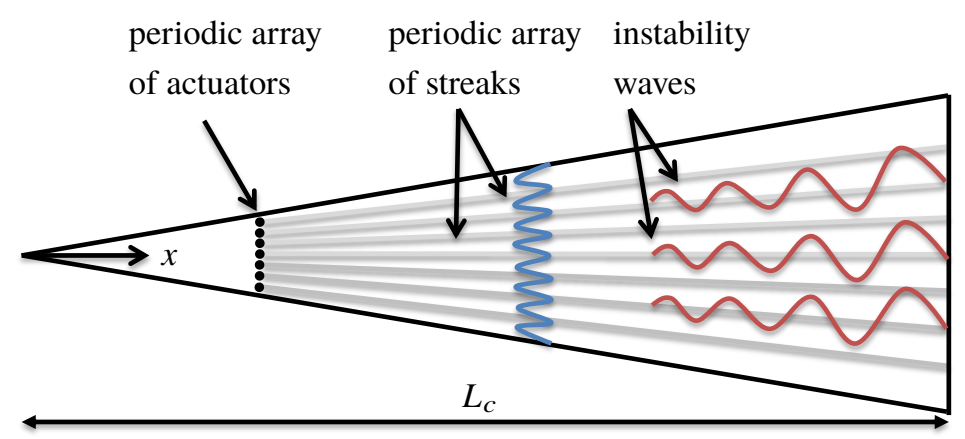

Fig. 1 Sketch (side view) of the cone illustrating the present conceptual configuration. The wake of the periodic array of actuators generate the periodic array of streaks that modulate the instability waves.

conditions.

To that end, we study the effects of the wake of one or more periodic arrays of wall-mounted vortex generators on the dominant instability waves in axisymmetric or two-dimensional boundary layers at hypersonic Mach numbers, i.e., the Mack-mode instabilities. Figure 1 shows a schematic of the flow configuration considered in this work. The geometry is a $7^{\circ}$ half-angle circular cone with $r_{n}^{*}=2.5 \mathrm{~mm}$ nose radius and $L_{c}^{*}=2.0 \mathrm{~m}$ length. The freestream parameters $\left(M_{\infty}=5.3, R e_{\infty}=13.42 \times 10^{6} \mathrm{~m}^{-1}, T_{\infty}^{*}=201.4 \mathrm{~K}\right)$ are selected to match the flow conditions of the HIFiRE-1 flight experiment during the ascent phase at time equal to $21.5 \mathrm{~s}$ [30]. The laminar boundary-layer flow is computed by solving the full Navier-Stokes equations. The good correlation between experimental measurements and theoretical predictions based on the parabolized stability equations (PSE) has confirmed that laminar-turbulent transition in this flow is driven by the modal growth of planar Mack-mode instabilities [31]. The perturbed three-dimensional boundary layer is used as a basic state for the subsequent modal instability analysis by means of the plane-marching PSE.

The paper is organized as follows. $\$$ II provides a summary of the plane-marching PSE method. The results are presented in \$III. First, the basic state and instability characteristics of the unperturbed boundary-layer flow are analyzed. Second, the DNS solution of the perturbed, three-dimensional boundary-layer flows for two cases with one and two arrays of vortex generators is shown. Subsequently, the plane marching PSE are used to examine the instability characteristics of the Mack-mode waves as well as the streak instabilities of the perturbed basic states. Finally, summary and concluding remarks are presented in $\$ \mathrm{IV}$

\section{Theory}

This section introduces the methodology behind the instability analysis described in this paper. The procedure closely follows the study of Paredes et al. [24] that analyzed the interactions between the Mack-mode waves and finite-amplitude optimal growth streaks in the same hypersonic boundary-layer flow as that analyzed herein. The main difference between the two studies corresponds to the nature of stationary streaks. Unlike the optimal growth streaks from the previous work that may not be easily realizable in practice, the present work focuses on realizable streaks due to one or more arrays of wall-mounted vortex generators (VGs). Direct numerical simulations (DNS) are used to compute the three-dimensional, azimuthally-periodic, boundary-layer flow perturbed by VG array(s). Similar to Paredes et al. [24], the modal instability characteristics of the perturbed three-dimensional boundary-layer flow are studied by using the linear form of plane-marching PSE.

\section{A. Plane-marching PSE}

The linear, plane-marching PSE are used to predict the linear amplification characteristics of both modulated Mack-mode waves and the streak instability waves sustained by finite amplitude streaks induced by the VGs. The initial disturbance profiles for the plane-marching PSE are obtained using a partial-differential-equation (PDE) based two-dimensional eigenvalue problem (EVP). In the plane-marching PSE context, the perturbations to the streak have the form

$$
\tilde{\mathbf{q}}(\xi, \eta, \zeta, t)=\hat{\mathbf{q}}(\xi, \eta, \zeta) \exp \left[\mathrm{i}\left(\int_{\xi_{0}}^{\xi} \alpha\left(\xi^{\prime}\right) \mathrm{d} \xi^{\prime}-\omega t\right)\right] .
$$


A more detailed description of the PDE-based two-dimensional EVP and plane-marching PSE methodologies are given by Paredes [32], Paredes et al. [33], and Paredes et al. [29].

The onset of laminar-turbulent transition is estimated using the logarithmic amplification ratio, the so-called $N$-factor, relative to the lower bound location $\xi_{l b}$ where the disturbance first becomes unstable,

$$
N=-\int_{\xi_{l b}}^{\xi} \alpha_{i}\left(\xi^{\prime}\right) \mathrm{d} \xi^{\prime}+1 / 2 \ln \left[\hat{E}(\xi) / \hat{E}\left(\xi_{l b}\right)\right]
$$

The energy norm of $\tilde{\mathbf{q}}$ was derived by Mack [34] and defined as

$$
E(\xi)=\frac{1}{L_{\zeta}} \int_{\zeta} \int_{\eta} \tilde{\mathbf{q}}(\xi, \eta, \zeta)^{H} \mathbf{M} \tilde{\mathbf{q}}(\xi, \eta, \zeta) h_{1} h_{3} \mathrm{~d} \eta \mathrm{d} \zeta,
$$

where $h_{1}$ and $h_{3}$ are the metric factors associated with the streamwise and azimuthal curvatures, respectively, the superscript $H$ denotes conjugate transpose, and $\mathbf{M}$ is the energy weight matrix equal to

$$
\mathbf{M}=\operatorname{diag}\left[\frac{\bar{T}(\xi, \eta)}{\gamma \bar{\rho}(\xi, \eta) M^{2}}, \bar{\rho}(\xi, \eta), \bar{\rho}(\xi, \eta), \bar{\rho}(\xi, \eta), \frac{\bar{\rho}(\xi, \eta)}{\gamma(\gamma-1) \bar{T}(\xi, \eta) M^{2}}\right] .
$$

Accordingly, we assume that transition onset is likely to occur when the peak $N$-factor reaches a specified value. Similarly, the nonparallel growth rate based on the Mack's energy norm $E$ is defined as

$$
\sigma=\frac{\mathrm{d} N}{\mathrm{~d} \xi}
$$

\section{B. Spatial discretization and boundary conditions}

The plane-marching PSE are integrated along the streamwise coordinate by using second-order backward differentiation. A constant step of $\Delta R=2$, where $R=\sqrt{R e_{\xi}}$, along the streamwise direction is used. Finite differences [35, 36] (FD-q) of sixth-order are used for discretization of the wall-normal coordinate with $N_{\eta}=241$ points. The nodes are clustered toward the wall [36]. No-slip, isothermal boundary conditions are used at the wall, i.e., $\hat{u}=\hat{v}=\hat{w}=\hat{T}=0$. The amplitude functions are forced to decay at the farfield boundary by imposing the Dirichlet conditions $\hat{\rho}=\hat{u}=\hat{w}=\hat{T}=0$. The farfield boundary coordinate is set just below the shock layer. The azimuthal direction is discretized with Fourier collocation points. Depending on the amplitude of the optimal growth perturbation, the number of azimuthal points is $N_{\zeta}=64$ per streak azimuthal wavelength, $\lambda_{S T}=2 \pi / m_{S T}$, where $m_{S T}$ denotes the streak wavenumber. However, for improved computational efficiency, some of the calculations were also performed by using a nonperiodic finite-difference discretization (FD-q) of sixth-order along the azimuthal direction. With the nonperiodic scheme, only one half of $\lambda_{S T}=2 \pi / m_{S T}$, where $m_{S T}$ denotes the streak wavenumber, needs to be discretized and the boundary conditions at $\zeta=0$ and $\zeta=\lambda_{S T} / 2$ determine the nature of the instability mode, i.e., whether the disturbance is sinuous (S) or varicose (V) type. Note that fundamental disturbances correspond to $m=m_{S T}$, whereas subharmonic disturbances analyzed herein correspond to $m=1 / 2 m_{S T}$ and $m=3 / 2 m_{S T}$. The azimuthal boundary conditions used in each case are explained in Paredes et al. [24].

The number of discretization points in all three directions and the wall-normal domain size were varied to ensure that the relevant flow quantities were insensitive to further improvement in grid resolution and enlargement of the domain size. Verification of the present plane-marching PSE module against line-marching PSE and DNS results is shown in Refs. [33, 37].

\section{Results}

We begin the presentation of numerical results with the axisymmetric boundary layer over a nearly sharp, $7^{\circ}$ half-angle circular cone at zero degrees angle of attack in a hypersonic free stream. The details of the basic state and its modal instability characteristics are introduced first. Then, the solutions for perturbed boundary-layer flows due to wall-mounted VGs are presented. Finally, the instability characteristics of the perturbed flows and the overall effects of the VG-induced streaks on the estimated transition onset location are analyzed. 

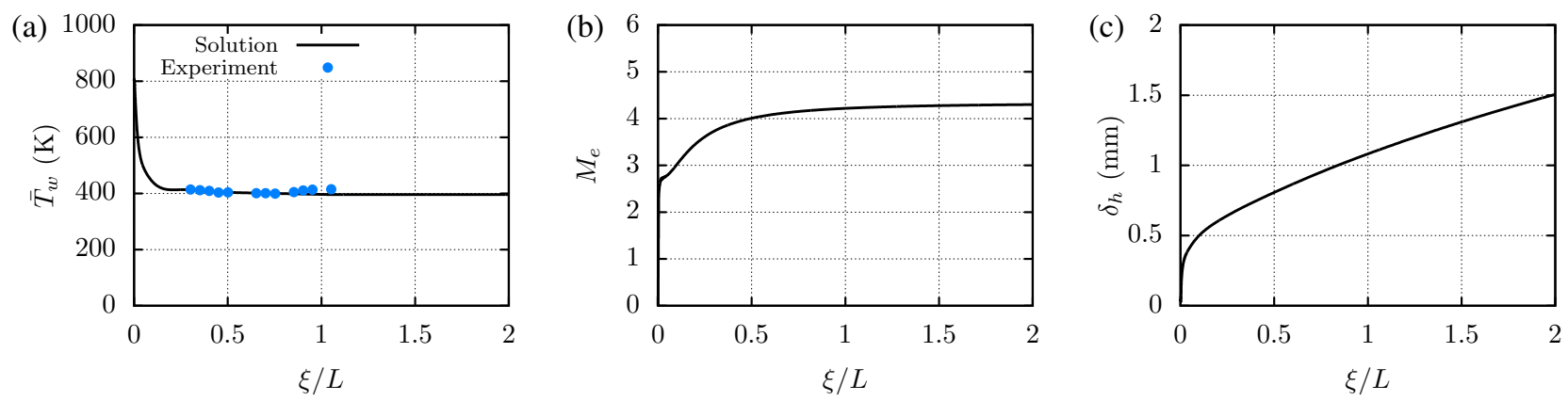

Fig. 2 Streamwise evolution of (a) wall temperature, (b) edge Mach number, and (c) boundary layer thickness. The wall temperature values measured in the flight experiments are included.

\section{A. Unperturbed boundary-layer flow}

\section{Basic state solution}

The flow configuration of interest corresponds to a $7^{\circ}$ half-angle, circular cone at zero degrees angle of attack in a hypersonic free stream. The length of the nearly sharp cone is $L_{c}^{*}=2.0 \mathrm{~m}$, and the nose radius is $r_{n}^{*}=2.5 \mathrm{~mm}$, so that the front half of the cone matches the HIFiRE-1 geometry. The extended cone length is used in the present investigation to assess the extent of potential delay in laminar-turbulent transition due to the streaks. The basic state corresponding to the laminar boundary-layer flow over the cone surface is computed by using a second-order accurate algorithm as implemented in the finite-volume compressible Navier-Stokes flow solver VULCAN-CFD* [38]. The VULCAN-CFD solution is based on the full Navier-Stokes equations and uses the solver's built-in capability to iteratively adapt the computational grid to the shock. Sutherland's law is assumed for bulk viscosity.

The freestream conditions are selected to replicate those of the HIFiRE-1 flight experiment at time equal to $21.5 \mathrm{~s}$ during the ascent phase [30], i.e., Mach 5.30 flow with a unit Reynolds number of $13.42 \times 10^{6} \mathrm{~m}^{-1}$, freestream temperature of $T_{\infty}^{*}=201.4 \mathrm{~K}$ and a prescribed surface temperature distribution that corresponds to a wall-to-adiabatic temperature ratio of approximately 0.35 over most of the vehicle [31]. Figure 2(a) shows the prescribed distribution of surface temperature along the length of the cone. As explained by Li et al. [31], this temperature distribution was obtained by combining the results of thermal analysis based on axisymmetric, finite-element calculations using the U.S. Air Force Research Laboratory (AFRL) TOPAZ code [39] and the experimental data based on thermocouple measurements [30]. The edge Mach number $M_{e}$ and boundary layer thickness $\delta_{h}$, which is defined as the wall-normal position where $h_{t} / h_{t, \infty}=0.995$, where $h_{t}$ is the total enthalpy, are plotted in figures 2 (b) and 2(c), respectively.

The computational grid has 865 points in the streamwise direction and 513 points in the wall normal direction. A minimum of 120 points is clustered next to the cone surface to resolve the thickness of the boundary layer. This grid resolution is based on the work of Li et al. [31], who computed the laminar flow over the HIFiRE-1 geometry at selected instants of times during the flight experiment by using the VULCAN-CFD flow solver. They also performed a grid-convergence test by doubling the number of points in each directions, and furthermore, verified the results by comparing the solution with that computed with a different Navier-Stokes solver in the form of the CFL3D code [40].

In what follows, freestream values are used as the reference values for nondimensionalization. The reference length scale is defined as $\delta=\sqrt{L v / u_{\infty}}$, where $L^{*}=1.0 \mathrm{~m}$. The computational coordinates, $(\xi, \eta, \zeta)$, correspond to an orthogonal, body-fitted coordinate system, where $\xi$ denotes the streamwise coordinate measured along the cone surface, $\eta$ is the surface-normal coordinate, and $\zeta$ is measured along the azimuthal direction. The relevant metric factors are defined as

$$
\begin{aligned}
& h_{\xi}=1+\kappa \eta, \\
& h_{\zeta}=r_{b}+\eta \cos (\theta),
\end{aligned}
$$

where $\kappa$ denotes the streamwise curvature, $r_{b}$ is the local radius, and $\theta$ is the local half-angle along the axisymmetric surface, i.e., $\sin (\theta)=\mathrm{d} r_{b} / \mathrm{d} \xi$. For the present straight circular cone (with exception of the nose region that is not included in this analysis), $\kappa \equiv 0$ and $\theta$ is the cone half-angle equal to $7^{\circ}$.

\footnotetext{
*visit http://vulcan-cfd.larc.nasa.gov for further information about the VULCAN-CFD solver
} 

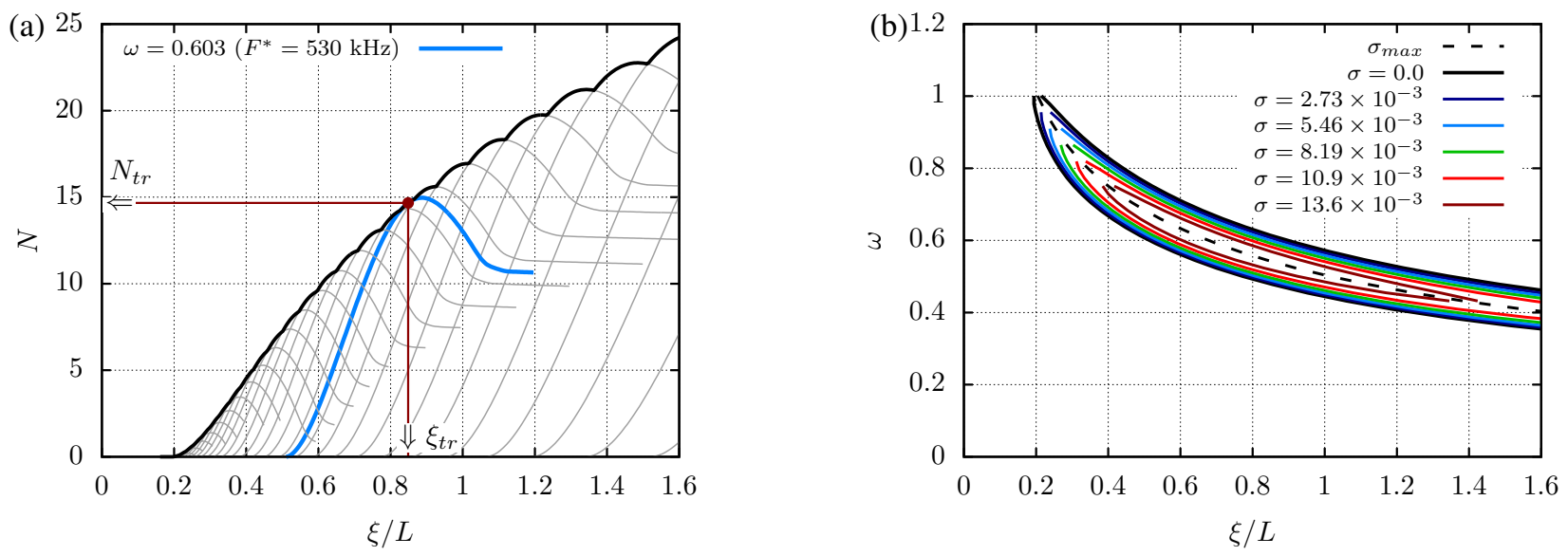

Fig. 3 (a) $N$-factors and (b) growth-rate isolines of planar Mack-mode disturbances in the unperturbed boundary layer. The thick blue line in (a) denotes the mode that reaches largest $N$-factor value $\left(N_{t r}\right)$ at the experimentally measured transition location $\left(\xi_{t r}\right)$ and corresponds to a disturbance frequency of $\omega=0.603$.

\section{Modal instability characteristics}

Experimental measurements and theoretical predictions based on PSE have confirmed that laminar-turbulent transition in this flow is driven by the modal growth of planar Mack-mode instabilities [31]. To establish the transition behavior in the absence of stationary streak perturbations, the instability of the unperturbed flow was examined by using PSE. The onset of laminar-turbulent transition in the unperturbed boundary-layer flow was estimated on the basis of $N$-factor evolution corresponding to the planar Mack modes computed with the PSE. For the conditions of the experiment [30], transition onset in the unperturbed cone boundary layer was measured to occur near $\xi_{t r} / L=0.85 \mathrm{~m}$. Figure 3 a) shows that the peak $N$-factor at the measured transition location corresponds to $N_{t r}=14.7$, which is first reached by a planar Mack-mode disturbance with a frequency of $\omega=0.603$. Figure 3(b) shows the growth-rate isolines of planar Mack-mode instabilities. The neutral stability curve corresponds to the black line with $\sigma=0.0$. Neither planar nor oblique first-mode instabilities were found to be unstable in the present boundary-layer flow because of the low surface temperature relative to the adiabatic temperature.

\section{B. Perturbed boundary-layer flow}

\section{Basic state solution}

The axisymmetric boundary-layer flow studied in section III.A is perturbed via arrays of axially localized roughness elements centered at selected axial stations. The roughness array is comprised of azimuthally equispaced pairs of smooth-shaped VGs with an elliptical platforms. Nominally, the height of the VGs increases linearly along the length of the VG; however, the height distribution is tapered on all sides to achieve a smooth shape. The three-dimensional, azimuthally-periodic, laminar boundary-layer flow over the cone surface with arrays of wall-mounted VGs is computed by using a high-order DNS solver that was recently used by Li et al. [41] to simulate the excitation of stationary crossflow vortices behind an array of roughness elements mounted on a yawed cone. A detailed description of the governing equations and their numerical solution is given by $\mathrm{Wu}$ and Martin [42]. The inviscid fluxes from the governing equations are computed using a seventh-order wighted essentially nonoscillatory finite-difference WENO scheme introduced by Jiang and Shu [43]; the present scheme also allows the use of limiters that have been optimized to reduce the numerical dissipation. Both an absolute limiter on the WENO smoothness measurement and a relative limiter on the total variation are employed simultaneously during the simulation. The viscous fluxes are discretized using a fourth-order central difference scheme and time integration is performed using a third-order low-storage Runge-Kutta scheme [44].

The computational grid is based on the grid used for the unperturbed solution, although the grid was clustered near the roughness location. The final grids have 983 points and 2288 points in the streamwise direction for cases I and II, respectively. For both cases, the computational domain along the wall normal and azimuthal directions has been discretized with 257 and 192 points, respectively. The grid resolution was checked for convergence by comparing the instability results to those obtained on a basic state solution with a coarser grid of half the number of points in every 
direction.

The VGs are designed on the basis of the earlier analysis by Paredes et al. [24], wherein the interaction between stationary streaks undergoing optimal, nonmodal growth and the Mack mode waves in the present hypersonic boundarylayer flow was analyzed. The geometrical details of the VGs employed in both of the cases examined herein are specified in Table 1. The first array of VGs is located at $\xi_{V G} / L=0.6059 \mathrm{~m}$, where the $\mathrm{N}$-factor value corresponding to the envelope of Mack modes waves in the unperturbed boundary layer is approximately $2 / 3$ times the $\mathrm{N}$-factor value correlating with the onset of transition, i.e., $N=2 / 3 N_{t r}$ as shown in figure 3 . The second array of VGs in the staged control case, i.e., case II of Table 1 is located at $\xi_{V G} / L=0.8376$. As shown subsequently in this paper, the latter location again corresponds to $N \approx 2 / 3 N_{t r}$, but where $N$ refers to the $\mathrm{N}$-factor envelope for the boundary layer flow modified by the upstream array of VGs (which corresponds to the baseline for the introduction of the downstream array of VGs). The azimuthal wavenumber of the VGs is set to $m_{S T}=320$. The choice of azimuthal wavenumber is consistent with the observation by Paredes et al. [24] that a wavelength to boundary-layer thickness ratio of $\lambda_{S T} r_{b} / \delta_{h} \in(1.4,2.2)$ is highly effective in stabilizing the Mack-mode instabilities.

Table 1 Geometrical properties of the studied cases. The azimuthal wavenumber is set to $m_{S T}=320$. The lateral angle of the second array of Case II is increased to keep the same lateral blockage as the first stage.

\begin{tabular}{ccrrrrrrr} 
Case & Array & $\xi_{V G} / L$ & $\delta_{h}^{*}[\mathrm{~mm}]$ & $\lambda_{S T} r_{b} / \delta_{h}$ & $k / \delta_{h}$ & $a_{V G} / \delta_{h}$ & $b_{V G} / \delta_{h}$ & $\phi\left[^{\circ}\right]$ \\
\hline I & 1 & 0.6059 & 0.8708 & 1.711 & 0.5053 & 2.010 & 0.1315 & 3.017 \\
\hline \multirow{2}{*}{ II } & 1 & 0.6059 & 0.8708 & 1.711 & 0.4674 & 2.679 & 0.1315 & 3.017 \\
& 2 & 0.8376 & 0.9995 & 2.045 & 0.4310 & 2.745 & 0.1585 & -3.547 \\
\hline
\end{tabular}

Figures 4(a) and 4 b) display three-dimensional views of the flow field in cases I and II, respectively, from Table 1 for a single azimuthal wavelength. The surface of the cone with the VG pairs is shown in gray color. Isolines of Mach number values at selected streamwise stations, $\xi / L=(0.65: 0.10: 1.45)$ are also shown. The disturbances induced by the VGs evolve into streamwise elongated streaks. The counter orientation of the downstream array of VGs with respect to the upstream array in case II yields an azimuthal phase shift of the streak shape. Figure 5 shows the axial evolution of nondimensional streak amplitude based on the streamwise velocity, where the streak amplitude, $A s_{u}(\xi)$, is defined by Fransson et al. [45] as

$$
A s_{u}(\xi)=\frac{1}{2 u_{\infty}} \max _{\eta}\left[\max _{\zeta}(\bar{u}(\xi, \eta, \zeta))-\min _{\zeta}(\bar{u}(\xi, \eta, \zeta))\right] .
$$

Both curves show a rapid rise to a narrow peak just behind the VG array at $\xi_{V G} / L=0.6059$, but the streak amplitudes exhibit a region of nonmodal growth after that initial peak. The highest streak amplitude is slightly larger in case I because the VG height, $k$, was slightly reduced for case II in order to minimize the potential for transition due to streak instabilities. The addition of a second array of VGs in case II yields higher streak amplitudes than case I at downstream axial locations $\left(\xi_{V G} / L>0.8376\right)$. The streak amplitude evolution in case II also indicates a slower rise to its downstream peak in comparison with the rather rapid rise observed for the upstream peak, perhaps due to the distance required for the flow to adjust to the reversed orientation of the downstream array of VGs. Although not shown here, a configuration identical to case II but with the same orientation of both VG arrays was found to result in a stronger peak in the streak amplitude after the second array.

\section{Modal instability characteristics}

The instability characteristics of the perturbed, streaky boundary-layer flow are examined next. The plane-marching PSE are used to monitor the evolution of the Mack-mode (MM) waves, which are modulated by the presence of the azimuthally periodic streaks, and streak instabilities (SI) that can become the most amplified modes and dominate the process of laminar-turbulent transition. The plane-marching PSE require a slow spatial variation along the streamwise direction of the basic state variables. To facilitate the marching of the plane-marching PSE, short segments of the axial domain corresponding to the VG arrays are removed from the computed basic state and substituted by a smooth interpolation of the flow variables across the end points of these segments. The effect of instability wave scattering by the VGs is not accounted for in the present work, although it is expected to be small because of the short length of the VGs as seen from the geometry details in Table 1. Paredes et al. [24] studied the interaction of oblique and planar Mack-mode waves with finite-amplitude optimal streaks and concluded that the planar Mack mode dominates the instability characteristics of both the unperturbed and the perturbed boundary-layer flows, as long as the streak 
(a)
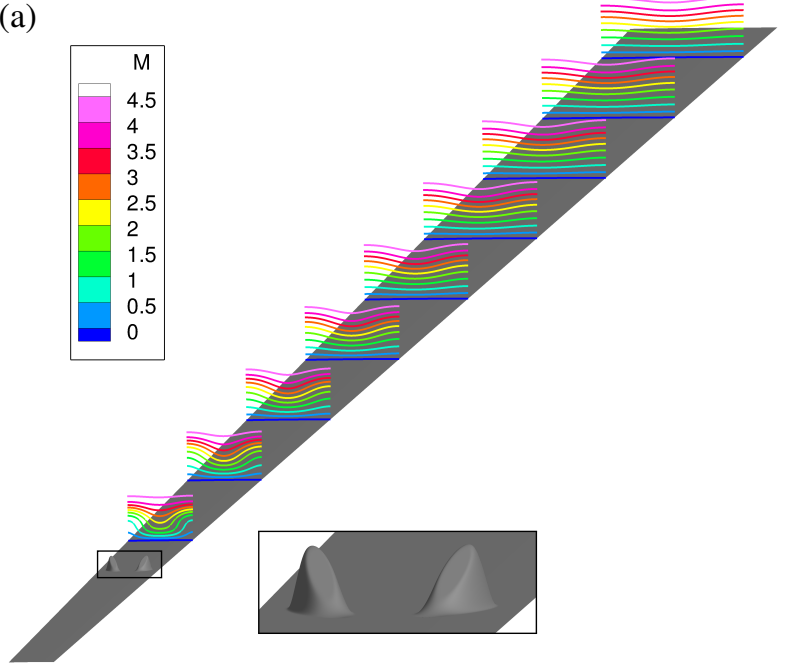

(b)

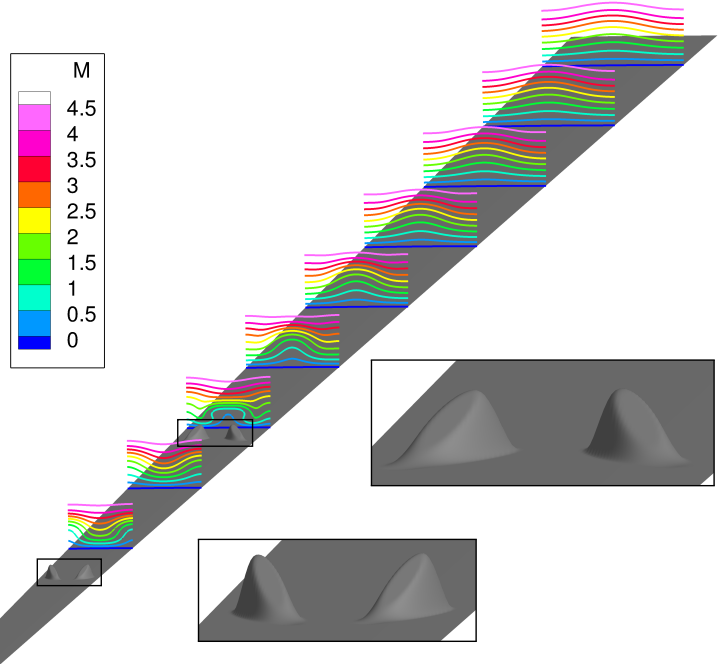

Fig. 4 Three-dimensional view of a single azimuthal wavelength of the modulated hypersonic boundary-layer flows corresponding to cases I and II. The zoomed images of the VG pairs are included. The wall is shown in gray color. Isolines of Mach number values are shown at selected streamwise locations.

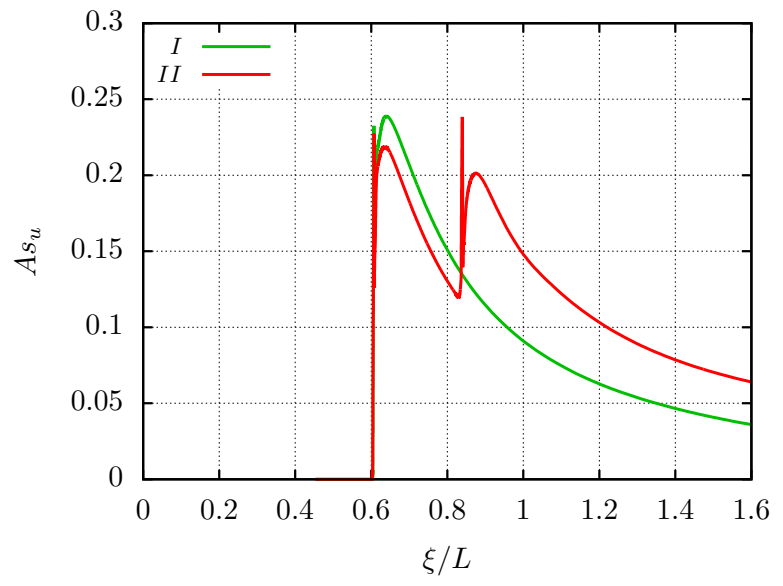

Fig. 5 Evolution of streak amplitude, $A s_{u}$, for the perturbed boundary-layer flows of cases I and II. 

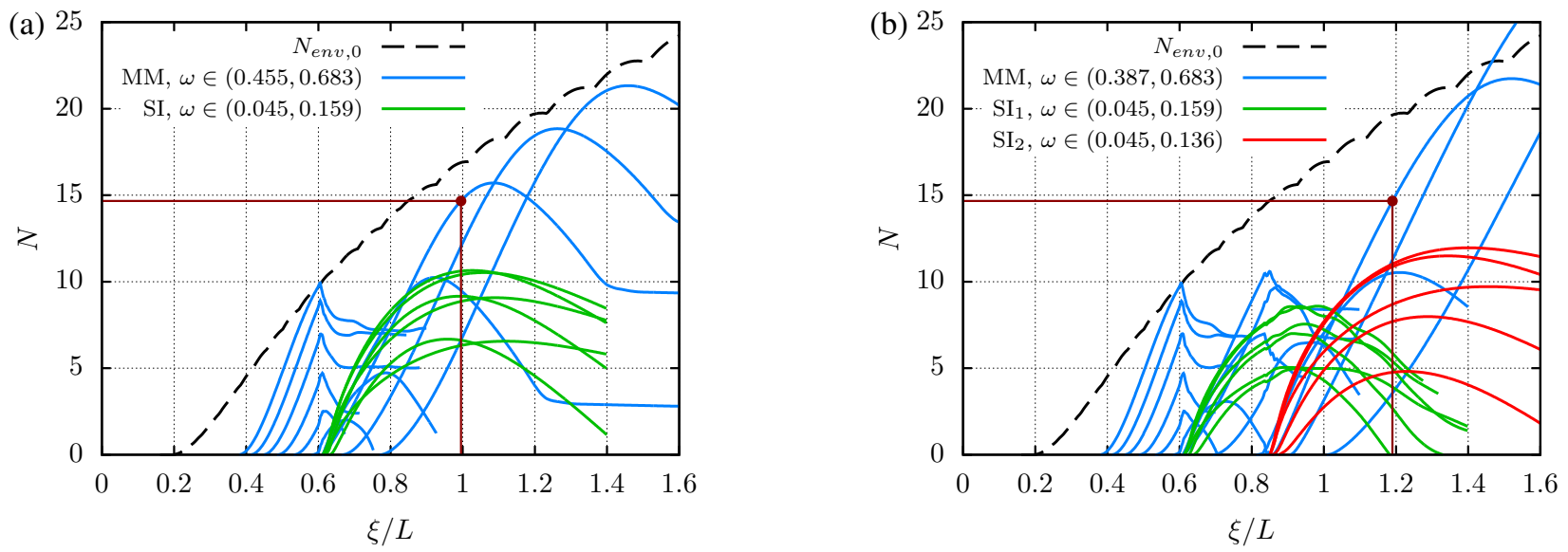

Fig. $6 N$-factor curves for planar Mack-mode disturbances (MM) and sinuous, subharmonic streak instability modes (SI) for (a) case I with one array of VGs at $\xi_{V G} / L=0.6059$ and (b) case II with two arrays of VGs at $\xi_{V G, 1} / L=0.6059$ and $\xi_{V G, 2} / L=0.8376$. The relevant frequency ranges are selected for each disturbance. The dark-red, solid horizontal and vertical lines denote the transition $\mathrm{N}$-factor and corresponding transition location.

amplitudes are not large enough for the shear layer instabilities sustained by those streaks to cause transition onset. Based on those findings, the growth of nominally planar Mack-mode waves is analyzed next. Paredes et al. [24] have also observed that the subharmonic, sinuous mode of streak instability is the most unstable mode for moderate streak amplitudes and, therefore, has the potential to play an important role during the transition process.

The overall effect of the VG-induced streaks on the instability characteristics of the hypersonic boundary-layer flow is shown in figures 6(a) and 6(b) for cases I and II, respectively. These figures show the $\mathrm{N}$-factor curves of the nominally planar Mack modes (MM) and subharmonic sinuous streak instability modes (SI). As seen from figure 6(a), the MM modes that grow upstream of the VG array at $\xi_{V G} / L=0.6059$ become damped behind the array, yielding a substantial reduction $(\Delta N \approx 6)$ in the peak $N$-factor up to $\xi_{V G} / L \approx 0.75$. Other MM waves that become unstable behind the VG array can eventually achieve large $N$-factors, and in particular, the MM wave with $\omega=0.501$ reaches the transition $N$-factor, $N_{t r}=14.7$, at $\xi_{t r, I} / L=0.995$ for case I, which represents a transition delay of $17.2 \%$ with respect to the uncontrolled boundary-layer flow. Streak instability waves (i.e., SI modes) also become unstable downstream of the VG array, although the peak $N$-factor of these waves is equal to $N=10.65$ (reached by the SI mode with $\omega=0.114$ at $\xi / L=1.027$ ), which is relatively low compared to the $N$-factor value correlating with transition in the flight experiment.

The staged control in case II attempts to further delay the transition location by introducing a second array of VGs to reinforce the streak amplitudes sufficiently farther downstream from the first VG array. The orientation of the second VG array is intentionally reversed with respect to the upstream array. Because of the resulting phase shift in the azimuthal velocity distribution associated with the streaks, the subharmonic streak instability modes introduced by the upstream VG array, $S I_{1}$, become damped behind the second VG array at $\xi_{V G, 2} / L=0.8376$ as shown in figure 6(b). The downstream array of VGs cannot prevent the subharmonic, sinuous streak instabilities, but the $N$-factors of those modes remain below the $N$-factor envelope for the MM waves. In this case, the SI modes achieve a peak $N$-factor of $N=11.96$, which is achieved at $\xi / L=1.4$ by the wave corresponding to a frequency of $\omega=0.0683$. However, the Mack mode wave with $\omega=0.432$ reaches $N_{t r}=14.7$ at an upstream station, $\xi_{t r, I I} / L=1.190$, and therefore, the resulting transition delay in case II is estimated to be $40.2 \%$ with respect to the transition location for the unperturbed boundary-layer flow. We expect that a second array of VGs with the same orientation as the upstream array would results in a further destabilization of the streak instabilities, and hence, a coaligned set of VG arrays is likely to be less effective in delaying transition than the set with alternating orientations as in case II.

Overall, the above results indicate a potential increase in laminar flow length of approximately $40 \%$ of the laminar flow region in the unperturbed case. Considering that the ratio of local skin friction coefficients for turbulent and laminar flows is in the range of $c_{f, \text { tur }} / c_{f, \text { lam }} \in(5,7)$ [46], the maximum reduction in skin friction drag relative to the unperturbed flow over the present test article with a length Reynolds number of $\operatorname{Re}_{L_{c}}=26.84 \times 10^{6}$ would be around $10 \%$ for case I and $23 \%$ for case II at the flow conditions of interest $\left(M_{\infty}=5.3, R e_{\infty}=13.42 \times 10^{6} \mathrm{~m}^{-1}\right.$, $T_{w} / T_{w, a d}=0.35$ ) and with a transition threshold of $N=14.7$. Based on Reynolds analogy, comparable reduction may 
also be expected in the total heat load experienced by the cone surface.

\section{Summary and concluding remarks}

The present paper investigates the potential of realizable, stationary streaks undergoing nonmodal growth to delay the laminar-turbulent transition onset in a high-speed boundary-layer flow over a $7^{\circ}$ half-angle cone at zero degrees angle of attack. The flow conditions $\left(M_{\infty}=5.3, R e_{\infty}=13.42 \times 10^{6} \mathrm{~m}^{-1}\right.$, and $\left.T_{\infty}^{*}=201.4 \mathrm{~K}\right)$ correspond to the HIFiRE-1 flight experiment during the ascent phase at time equal to $21.5 \mathrm{~s}$ [30]. The compressible Navier-Stokes equations are used to obtain the laminar flow solution of both, the unperturbed, two-dimensional boundary-layer flow and the three-dimensional boundary-layer flow perturbed by wall-mounted arrays of vortex generators (VGs). In the absence of the control devices, the good correlation between experimental measurements and theoretical predictions based on the parabolized stability equations (PSE) confirmed that laminar-turbulent transition in this flow is driven by the modal growth of planar Mack-mode instabilities [31]. The VGs are designed on the basis of the earlier analysis by Paredes et al. [24], wherein the interaction between stationary streaks undergoing optimal, nonmodal growth and the Mack-mode waves in the same hypersonic boundary-layer flow was analyzed. The linear stability characteristics of the perturbed, boundary-layer flows are studied by using the linear form of the plane-marching PSE. Two configurations are studied, one case with a single array of VGs and a second case with two separate arrays with opposite orientations. The latter case was designed to investigate staged control of Mack-mode instabilities while simultaneously reducing the amplification of streak instabilities resulting from the control devices. The instability analysis results for the single VG array configuration demonstrate the potential of realizable streaks induced by means of wall-mounted devices to reduce the peak amplification of boundary-layer instabilities, indicating the possibility of a transition delay of $17 \%$ with respect to the uncontrolled case. The results for the configuration with two separate arrays with opposite orientations confirm that the staged control is more effective in delaying the onset of transition, yielding an increase of up to $40 \%$ in the laminar flow region via further reduction in the peak amplification factor of Mack-mode instabilities while keeping the amplification of streak instabilities below the transition threshold. We note that the amount of transition delay is calculated by assuming that the $N$-factor values correlating with transition onset remain similar in both unperturbed and perturbed cases. However, even if the transition $N$-factor threshold is reduced by 1 in the presence of the VG arrays, the (reduced) delay in transition would still be quite significant, namely, $13 \%$ for the configuration with one VG array and $37 \%$ for the staged control case.

Detailed laboratory experiments are required to establish the effect of streaks, if any, on the $N$-factor correlation. The effect of the wall-mounted devices on the generation (i.e., receptivity) of the unsteady modal instabilities is also not addressed in the present investigation. A study of both these aspects would represent an important extension of the present work. Furthermore, the analysis of the nonlinear breakdown for the baseline and controlled flows, the inclusion of additional VG arrays with alternate orientation to study the potential for improved transition delay, as well as the effects of scattering by multiple arrays on the instability wave evolution, especially on the nonlinear phase, will be considered in the future development of this concept.

\section{Acknowledgments}

This research was sponsored by the NASA Transformational Tools and Technologies (TTT) Project of the Transformative Aeronautics Concepts Program (TACP) under the Aeronautics Research Mission Directorate (ARMD). Two of the authors (MC and FL) were also supported by the Hypersonic Technology project in the course of this work. A portion of the computational resources supporting this work were provided by the NASA High-End Computing (HEC) Program through the NASA Advanced Supercomputing (NAS) Division at Ames Research Center.

\section{References}

[1] Mack, L., "Boundary Layer Linear Stability Theory," AGARD-R-709. Special Course on Stability and Transition of Laminar Flow, 1984, pp. 3.1-3.81.

[2] Klebanoff, P., "Effect of Free-Stream Turbulence on the Laminar Boundary Layer," Bull. Am. Phys. Soc., 16, $1323,1971$.

[3] Vermeersch, O., and Arnal, D., "Klebanoff-Mode Modeling and Bypass-Transition Prediction,” AIAA J., Vol. 48, No. 11, 2010, pp. 2491-2500. 
[4] Andersson, P., Brandt, L., Bottaro, A., and Henningson, D., "On the Breakdown of Boundary Layer Streaks," J. Fluid Mech., Vol. 428, 2001, pp. 29-60.

[5] Boiko, A., Westin, K., Klingmann, B., Kozlov, V., and Alfredsson, P., "Experiments in a Boundary Layer Subjected to Free Stream Turbulence. PART 2. The Role of TS-Waves in the Transition Process," J. Fluid Mech., Vol. 281, 1994, pp. $219-245$.

[6] Cossu, C., and Brandt, L., "Stabilization of Tollmien-Schlichting Waves by Finite Amplitude Optimal Streaks in the Blasius Boundary Layer," Phys. Fluids, Vol. 14, No. 8, 2002, pp. L57-L60.

[7] Bagheri, S., and Hanifi, A., "The Stabilizing Effect of Streaks on Tollmien-Schlichting and Oblique Waves: A Parametric Study,” Phys. Fluids, Vol. 19, 2007, pp. 078103-1-078103-4.

[8] Fransson, J., Talamelli, A., Brandt, L., and Cossu, C., "Delaying Transition to Turbulence by a Passive Mechanism," Phys. Rev. Lett., Vol. 96, 2006, p. 064501.

[9] Shahinfar, S., Sattarzadeh, S., Fransson, J., and Talamelli, A., "Revival of Classical Vortex Generators Now for Transition Delay," Phys. Rev. Lett., Vol. 109, 2012, p. 074501.

[10] James, C., "Boundary-Layer Transition on Hollow Cylinders in Supersonic Free Flight as Affected by Mach Number and a Screwthread Type of Surface Roughness," NASA TR-Memo-1-20-59A, 1959.

[11] Fujii, K., "Experiment of the Two-Dimensional Roughness Effect of Hypersonic Boundary-Layer Transition,” J. Spacecraft Rockets, Vol. 43, No. 4, 2006, pp. 731-738.

[12] Fong, K., Wang, X., and Zhong, X., "Numerical Simulation of Roughness Effect on the Stability of a Hypersonic Boundary Layer," Compt. Fluids, Vol. 96, 2014, pp. 350-367.

[13] Fong, K., Wang, X., Huang, Y., Zhong, X., McKiernan, G., Fisher, R., and Schneider, S., "Second Mode Suppression in Hypersonic Boundary Layer by Roughness: Design and Experiments,” AIAA J., Vol. 53, No. 10, 2015, pp. 3138-3143.

[14] Holloway, P., and Sterrett, J., "Effect of Controlled Surface Roughness on Boundary-Layer Transition and Heat Transfer at Mach Number of 4.8 and 6.0," NASA TR-D-2054, 1964.

[15] Choudhari, M., Li, F., and Edwards, J., "Stability Analysis of Roughness Array Wake in a High-Speed Boundary Layer," AIAA Paper 2009-0170, 2009.

[16] Paredes, P., De Tullio, N., Sandham, N. D., and Theofilis, V., "Instability Study of the Wake Behind a Discrete Roughness Element in a Hypersonic Boundary Layer," Instability and control of massively separated flows, edited by V. Theofilis and J. Soria, Springer International Publishing, 2015, pp. 91-96.

[17] Li, F., Choudhari, M., Chang, C.-L., Greene, P., and Wu, M., "Development and Breakdown of Görtler Vortices in High Speed Boundary Layers," AIAA Paper 2010-0705, 2010.

[18] Li, F., Choudhari, M., Paredes, P., and Duan, L., "Secondary Instability of Stationary Crossflow Vortices in Mach 6 Boundary Layer over a Circular Cone,” NASA TM-2015-218997, 2015.

[19] Choudhari, M., Li, F., Paredes, P., and Duan, L., "Computations of Crossflow Instability in Hypersonic Boundary Layers," AIAA Paper 2017-4300, 2017.

[20] Choudhari, M., Li, F., Paredes, P., and Duan, L., "Nonlinear Evolution and Breakdown of Azimuthally Compact Crossflow Vortex Pattern over a Yawed Cone," AIAA Paper 2018-1823, 2018.

[21] Ren, J., Fu, S., and Hanifi, A., "Stabilization of the Hypersonic Boundary Layer by Finite-Amplitude Streaks," Phys. Fluids, Vol. 28, 2016, pp. 024110-1-16.

[22] Paredes, P., Choudhari, M., and Li, F., "Transition Delay in Hypersonic Boundary Layers via Optimal Perturbations," NASA TM-2016-219210, 2016.

[23] Paredes, P., Choudhari, M., and Li, F., "Stabilization of Hypersonic Boundary Layers by Linear and Nonlinear Optimal Perturbations," AIAA Paper 2017-3634, 2017.

[24] Paredes, P., Choudhari, M., and Li, F., "Instaiblity Wave-Streak Interactions in a Hypersonic Boundary Layer at Flight Conditions," J. Fluid Mech., 2018 (In review).

[25] Schmid, P. J., “Nonmodal Stability Theory,” Annu. Rev. Fluid Mech., Vol. 39, 2007, pp. 129-162. 
[26] Paredes, P., Choudhari, M., and Li, F., "Nonlinear Transient Growth and Boundary Layer Transition," AIAA Paper 2016-3956, 2016.

[27] Paredes, P., Choudhari, M., and Li, F., "Transition Due to Streamwise Streaks in a Supersonic Flat Plate Boundary Layer," Phys. Rev. Fluids, Vol. 1, 2016, pp. 083601-1-23.

[28] Paredes, P., Choudhari, M., and Li, F., “Transient Growth and Streak Instabilities on a Hypersonic Blunt Body,” AIAA Paper 2017-0066, 2017.

[29] Paredes, P., Choudhari, M., and Li, F., "Instaiblity Wave-Streak Interactions in a Supersonic Boundary Layer," J. Fluid Mech., Vol. 831, 2017, pp. 524-553.

[30] Kimmel, R., Adamczak, D., Paull, A., Paull, R., Shannon, J., Pietsch, R., Frost, M., and Alesi, H., "HIFiRE-1 Ascent-Phase Boundary-Layer Transition,” J. Spacecraft Rockets, Vol. 52, No. 1, 2015, pp. 217-230.

[31] Li, F., Choudhari, M., Chang, C., Kimmel, R., Adamczak, D., and Smith, M., "Transition Analysis for the Ascent Phase of HIFiRE-1 Flight Experiment,” J. Spacecraft Rockets, Vol. 52, No. 5, 2015, pp. 1283-1293.

[32] Paredes, P., “Advances in Global Instability Computations: from Incompressible to Hypersonic Flow," Ph.D. thesis, Universidad Politécnica de Madrid, 2014.

[33] Paredes, P., Hanifi, A., Theofilis, V., and Henningson, D., "The Nonlinear PSE-3D Concept for Transition Prediction in Flows With a Single Slowly-Varying Spatial Direction,” Procedia IUTAM, Vol. 14C, 2015, pp. 35-44.

[34] Mack, L. M., “Boundary Layer Stability Theory,” Tech. Rep. 900-277, Jet Propulsion Lab., Pasadena, CA, 1969.

[35] Hermanns, M., and Hernández, J. A., "Stable High-Order Finite-Difference Methods Based on Non-Uniform Grid Point Distributions," Int. J. Numer. Meth. Fluids, Vol. 56, 2008, pp. 233-255.

[36] Paredes, P., Hermanns, M., Le Clainche, S., and Theofilis, V., "Order $10^{4}$ Speedup in Global Linear Instability Analysis using Matrix Formation,” Comput. Meth. Appl. Mech. Eng., Vol. 253, 2013, pp. 287-304.

[37] De Tullio, N., Paredes, P., Sandham, N., and Theofilis, V., "Roughness-Induced Instability and Breakdown to Turbulence in a Supersonic Boundary-Layer," J. Fluid Mech., Vol. 735, 2013, pp. 613-646.

[38] Litton, D., Edwards, J., and White, J., “Algorithmic Enhancements to the VULCAN Navier-Stokes Solver," AIAA Paper 2003-3979, 2003.

[39] Kimmel, R., Adamczak, D., Gaitonde, D., Rougeux, A., and Haynes, J., "HIFiRE-1 Boundary Layer Transition Experiment Design," AIAA Paper 2007-0534, 2007.

[40] Rumsey, C., Biedron, R., and Thomas, J., “CFL3D: Its History and Some Recent Applications,” NASA TM-112861, 1997.

[41] Li, F., Choudhari, M., Paredes, P., and Duan, L., "High-Frequency Instabilities of Stationary Crossflow Vortices in a Hypersonic Boundary Layer," Phys. Rev. Fluids, Vol. 1, 2016, pp. 053603-1-32.

[42] Wu, M., and Martin, M., "Direct Numerical Simulation of Supersonic Boundary Layer over a Compression Ramp," AIAA J., Vol. 45, No. 4, 2007, pp. 879-889.

[43] Jiang, G., and Shu, C., "Efficient Implementation of Weighted ENO Schemes," J. Comp. Phys., Vol. 126, No. 1, 1996, pp. $202-228$.

[44] Williamson, J., “Low-Storage Runge-Kutta Schemes,” J. Comp. Phys., Vol. 35, No. 1, 1980, pp. $48-56$.

[45] Fransson, J., Brandt, L., Talamelli, A., and Cossu, C., "Experimental and Theoretical Investigation of the Non-Modal Growth of Steady Streaks in a Flat Plate Boundary Layer," Phys. Fluids, Vol. 16, No. 10, 2004, pp. 3627-3638.

[46] Schlichting, H., Boundary Layer Theory, McGraw-Hill, 1979. 